Document downloaded from:

http://hdl.handle.net/10251/66348

This paper must be cited as:

Cordero Barbero, A.; Feng, L.; Magrenan, A.; Torregrosa Sánchez, JR. (2015). A new fourth-order family for solving nonlinear problems and its dynamics. Journal of Mathematical Chemistry. 53(3):893-910. doi:10.1007/s10910-014-0464-4.

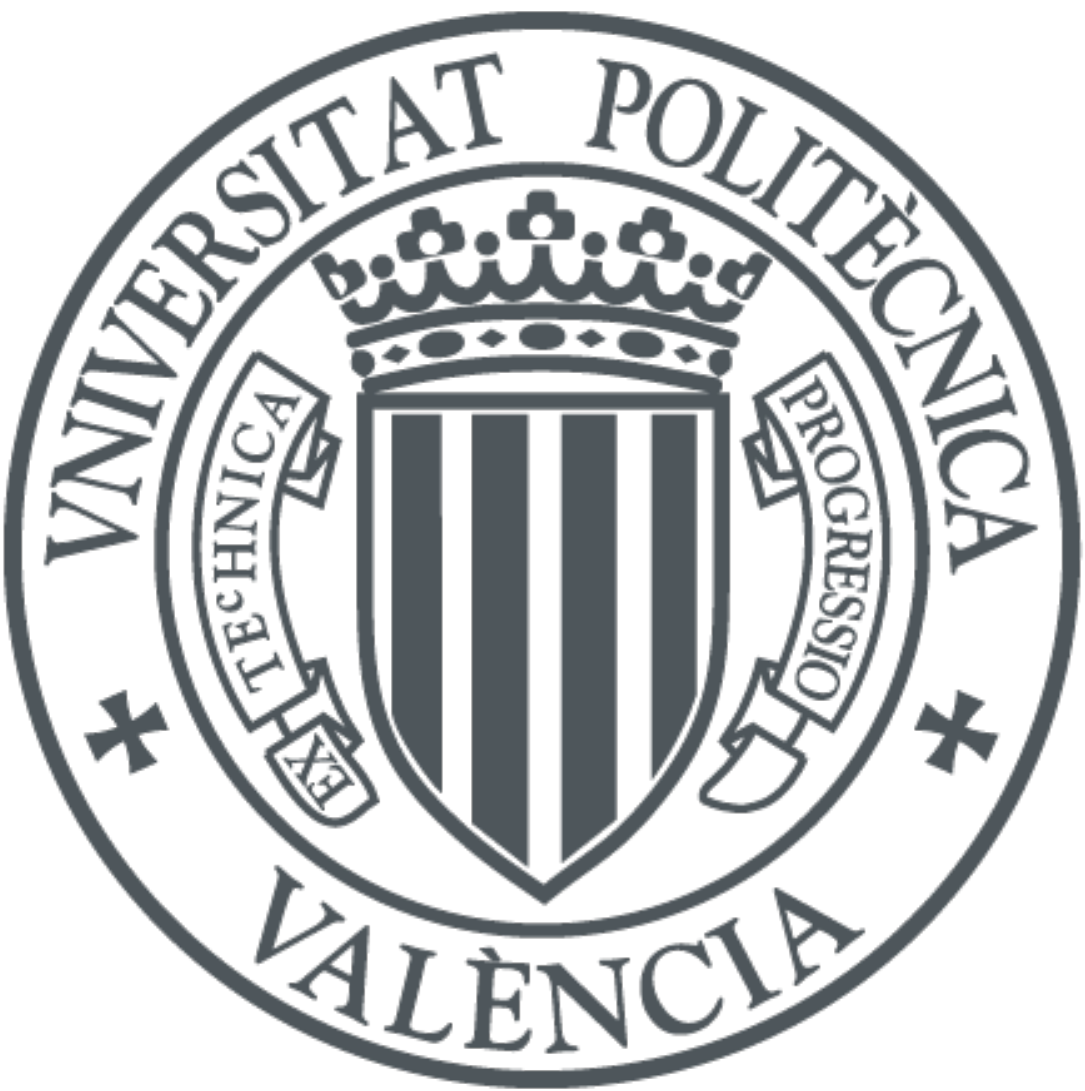

The final publication is available at

http://dx.doi.org/10.1007/s10910-014-0464-4

Copyright Springer Verlag (Germany)

Additional Information 


\title{
A new fourth-order family for solving nonlinear problems and its dynamics *
}

\author{
Alicia Cordero, Licheng Feng, Alberto Magreñán, Juan R. Torregrosa \\ Instituto de Matemáticas Multidisciplinar, Universitat Politècnica de València, 46022 València, Spain \\ Northwestern Polytechnical University, Xi'an 710072, Shaanxi Province, P.R.China \\ Departamento de TFG/TFM, Universidad Internacional de La Rioja (UNIR),26002 Logroño, La Rioja, Spain \\ acordero@mat.upv.es,fenglicheng1992@gmail.com,alberto.magrenan@gmail.com,jrtorre@mat.upv.es
}

\begin{abstract}
In this manuscript, a new parametric class of iterative methods for solving nonlinear systems of equations is proposed. Its fourth-order of convergence is proved and a dynamical analysis on low-degree polynomials is made in order to choose those elements of the family with better conditions of stability. These results are checked by solving the nonlinear system that arises from the partial differential equation of molecular interaction.
\end{abstract}

Keywords: Nonlinear systems, iterative methods, complex dynamics, parameter space, basins of attraction, stability.

MSC 2000: 65H05.

\section{Introduction}

In many branches of applied mathematics, chemistry, physics and engineering, efficient solvers of nonlinear problems are needed: in mass and heat transfer within porous catalyst particles, some boundary value problems for integro-differential equations appear that, after accurate discretization, Adomian decomposition or other techniques, yield to a nonlinear system of equations (see, for example [1] and [2]). Also nonlinear reactiondiffusion equations arise in autocatalytic chemical reactions (see [3]) or the analysis of the electronic structure of the hydrogen-atom in strong magnetic fields can be treated numerically, [4], by using iterative procedures for solving nonlinear problems. In fact, the numerical treatment of some specific chemical problems can help researchers to check their models of observable phenomena [5].

Efficient iterative methods are necessary for solving nonlinear Hammerstein integral equations arising from chemical phenomena. The following integral equation, called COSMO-RS (see [6, 7]), forms the basis for the conductor like screening model for real solvent which appeared in chemical phenomenon:

$$
\mu_{S}(\sigma)={ }_{R} T \ln \left[\int P_{S}\left(\sigma^{\prime}\right) \exp \left(-\frac{E_{i n t}\left(\sigma, \sigma^{\prime}\right)-\mu_{S}\left(\sigma^{\prime}\right)}{R T}\right) d \sigma^{\prime}\right],
$$

where $R$ is the gas constant, $T$ is the temperature and the term $E_{\text {int }}\left(\sigma, \sigma^{\prime}\right)$ denotes the interaction energy expression for the segments with screening charge density $\sigma$ and $\sigma^{\prime}$ respectively, the molecular interaction in solvent is $P_{S}(\sigma)$ and the chemical potential of the surface segments is described by $\mu_{S}(\sigma)$ which is to be determined. The resulting chemical potentials are the basis for other thermodynamic equilibrium properties such as activity co-efficients, solubility, partition co-efficients, vapor pressure and free energy of solvation (see $[8,9,10,11]$. Quantum chemical effects like group-group interaction, mesomeric effects and inductive effects also are incorporated into COSMO-RS by this approach.

\footnotetext{
*This research was supported by Ministerio de Ciencia y Tecnología MTM2011-28636-C02-\{01,02\} and Universitat Politècnica de València SP20120474.
} 
By using a particular discretization we can reduce the integral equation to algebraic nonlinear system and we can solve it by any iterative method.

The best known iterative scheme for solving a nonlinear system of equations $F(x)=0$ (being $F: D \subset \mathbb{R}^{n} \rightarrow$ $\left.\mathbb{R}^{n}\right), n \geq 1$ and the most used one, is Newton's method. Its iterative expression is

$$
x^{(k+1)}=x^{(k)}-\left[F^{\prime}\left(x^{(k)}\right)\right]^{-1} F\left(x^{(k)}\right), \quad k=0,1, \ldots
$$

where $F^{\prime}(x)$ denotes the Jacobian matrix associated to function $F$. In last years, some researchers in the area have developed higher order iterative procedures, trying to improve the efficiency and applicability of Newton's method. See, for example [12] and the references therein. Some concepts related with these aims are described in the rest of the section.

Firstly, we remember some known notions and results that we need in order to analyze the convergence of the developed methods.

Definition 1 Let $\left\{x^{(k)}\right\}_{k \geq 0}$ be a sequence in $\mathbb{R}^{n}$ convergent to $\alpha$. Then, convergence is said to be

(a) linear, if there exist $M, 0<M<1$, and $k_{0} \in \mathbb{N}$ such that

$$
\left\|x^{(k+1)}-\alpha\right\| \leq M\left\|x^{(k)}-\alpha\right\|, \forall k \geq k_{0} .
$$

(b) of order $p, p>1$, if there exist $M, M>0$, and $k_{0} \in \mathbb{N}$ such that

$$
\left\|x^{(k+1)}-\alpha\right\| \leq M\left\|x^{(k)}-\alpha\right\|^{p}, \forall k \geq k_{0} .
$$

In the numerical tests, we will use the concept of approximated computational order of convergence, that was introduced in [13] as follows

Definition 2 Let $\alpha$ be a zero of a function $F$ and suppose that $x^{(k-1)}, x^{(k)}$ and $x^{(k+1)}$ are three consecutive iterations close to $\alpha$. Then, the approximated computational order of convergence p estimates the theoretical order of convergence and can be calculated by means of the formula

$$
p \approx A C O C=\frac{\ln \left(\left\|x^{(k+1)}-x^{(k)}\right\| /\left\|x^{(k)}-x^{(k-1)}\right\|\right)}{\ln \left(\left\|x^{(k)}-x^{(k-1)}\right\| /\left\|x^{(k-1)}-x^{(k-2)}\right\|\right)} .
$$

In addition, in order to compare different methods, we will also use the efficiency index, $I=p^{1 / d}$, where $p$ is the order of convergence and $d$ is the total number of new functional evaluations, per iteration, required by the scheme. Related to this, Kung and Traub in [14] conjectured that the order of convergence of any iterative method without memory for solving nonlinear equations cannot exceed the bound $2^{d-1}$, (called the optimal order). This conjecture was defined only for scalar equations but it can be useful to classify the methods for systems and to select the most efficient ones. Ostrowski's method [15], Jarratt's scheme [16] and King's procedure [17] are some of optimal one-dimensional fourth order methods, being Jarratt's procedure specially useful for the multidimensional case. The notation that will be used in the proof of the main result of the convergence analysis was introduced in [18].

The dynamical behavior of the rational function obtained by applying the fixed point operator of an iterative method on a low degree polynomial gives us important information about its stability and reliability. In this context, recently different results have appeared in the literature (see for example [19, 20, 21, 22, 23, 24, 25]).

The rest of the paper is organized as follows: in Section 2, we introduce the parametric family of iterative methods and show its local order of convergence. A dynamical study of the parametric family is presented in Section 3, in order to see which is the relation between the stability of a method and the value of the parameter. In the numerical section, we apply different elements of our family for solving the nonlinear system resulting from the discretization of the equation of molecular interaction. Finally, some conclusions are established in the last section. 


\section{The proposed family and its convergence}

In this paper, we propose a one-parametric family of iterative methods, that can be used for $n \geq 1$, whose expression is

$$
\begin{aligned}
y^{(k)} & =x^{(k)}-\frac{2}{3}\left[F^{\prime}\left(x^{(k)}\right)\right]^{-1} F\left(x^{(k)}\right), \\
x^{(k+1)} & =x^{(k)}-\left(I-\frac{3}{4} A^{-1} B\right)\left[F^{\prime}\left(x^{(k)}\right)\right]^{-1} F\left(x^{(k)}\right), \quad k=0,1, \ldots
\end{aligned}
$$

where $u^{(k)}=\left[F^{\prime}\left(x^{(k)}\right)\right]^{-1}\left(F^{\prime}\left(y^{(k)}\right)-F^{\prime}\left(x^{(k)}\right)\right), A=I+\left(\frac{3}{2}+\beta\right) u^{(k)}, B=u^{(k)}+\beta u^{(k)^{2}}$ and $\beta$ is an arbitrary parameter.

A known method appear in this family when an specific value of the parameter is chosen; if $\beta=0$, Jarratt's scheme is obtained.

Theorem 1 Let $\alpha \in D$ be a zero of a sufficiently differentiable function $F: D \in \mathbb{R}^{n} \rightarrow \mathbb{R}^{n}, n \geq 1$, in a convex set $D$ with nonsingular Jacobian at $\alpha$, and let also $x^{(0)}$ be an initial approximation close enough to $\alpha$. For any real value of parameter $\beta$, the scheme defined in (2) provides fourth order of convergence, whose error equation is given by

$$
e^{(k+1)}=\left(\left(1-\frac{8}{3} \beta\right) C_{2}^{3}-C_{3} C_{2}+\frac{1}{9} C_{4}\right) e^{(k)^{4}}+O\left(e^{(k)^{5}}\right)
$$

where $C_{j}=\frac{1}{j !}\left[F^{\prime}(\alpha)\right]^{-1} F^{(j)}(\alpha), j=2,3, \ldots$ and $e^{(k)}=x^{(k)}-\alpha$.

Proof. By using Taylor expansion of $F\left(x^{(k)}\right)$ and $F^{\prime}\left(x^{(k)}\right)$ around $\alpha$,

$$
F\left(x^{(k)}\right)=F^{\prime}(\alpha)\left(e^{(k)}+C_{2} e^{(k)^{2}}+C_{3} e^{(k)^{3}}+C_{4} e^{(k)^{4}}+O\left(e^{(k)^{5}}\right)\right.
$$

and

$$
F^{\prime}\left(x^{(k)}\right)=F^{\prime}(\alpha)\left(I+2 C_{2} e^{(k)}+3 C_{3} e^{(k)^{2}}+4 C_{4} e^{(k)^{3}}+O\left(e^{(k)^{4}}\right)\right)
$$

Forcing $\left[F^{\prime}\left(x^{(k)}\right)\right]^{-1} F^{\prime}\left(x^{(k)}\right)=F^{\prime}\left(x^{(k)}\right)\left[F^{\prime}\left(x^{(k)}\right)\right]^{-1}=I$, we get

$$
\left[F^{\prime}\left(x^{(k)}\right)\right]^{-1}=\left(I+X_{2} e^{(k)}+X_{3} e^{(k)^{2}}+X_{4} e^{(k)^{3}}+O\left(e^{(k)^{4}}\right)\right)\left[F^{\prime}(\alpha)\right]^{-1},
$$

where $X_{1}=I$ and $X_{m}=-\sum_{j=2}^{m} j X_{m-j+1} C_{j}, m=2,3, \ldots$ Then, the error expression in the first step of the method is

$$
y^{(k)}-\alpha=\frac{1}{3} e^{(k)}+\frac{2}{3} C_{2} e^{(k)^{2}}-\frac{4}{3}\left(C_{2}^{2}-C_{3}\right) e^{(k)^{3}}+\frac{2}{3}\left(4 C_{2}^{3}-4 C_{2} C_{3}-3 C_{3} C_{2}+3 C_{4}\right) e^{(k)^{4}}+O\left(e^{(k)^{5}}\right) .
$$

Furthermore,

$$
F^{\prime}\left(y^{(k)}\right)=F^{\prime}(\alpha)\left(I+\frac{2}{3} C_{2} e^{(k)}+\frac{4}{3} C_{2}^{2}+\frac{1}{3} C_{3} e^{(k)^{2}}+Y_{4} e^{(k)^{3}}+O\left(e^{(k)^{4}}\right)\right),
$$

where $Y_{4}=-\frac{8}{3} C_{2}^{3}+\frac{8}{3} C_{2} C_{3}+\frac{4}{3} C_{3} C_{2}+\frac{4}{27} C_{4}$.

We get the Taylor expansion of $u^{(k)}$ by using (3) and (4):

$$
\begin{aligned}
u^{(k)} & =\left[F^{\prime}\left(x^{(k)}\right)\right]^{-1}\left[F^{\prime}\left(y^{(k)}\right)-F^{\prime}\left(x^{(k)}\right)\right] \\
& =-\frac{4}{3} C_{2} e^{(k)}+\left(4 C_{2}^{2}-\frac{8}{3} C_{3}\right) e^{(k)^{2}}+\left(-\frac{32}{3} C_{2}^{3}+8 C_{2} C_{3}+\frac{16}{3} C_{3} C_{2}-\frac{104}{27} C_{4}\right) e^{(k)^{3}}+O\left(e^{(k)^{4}}\right) .
\end{aligned}
$$

So, we can obtain

$$
\begin{aligned}
{\left[I+\left(\frac{3}{2}+\beta\right) u^{(k)}\right]^{-1}=} & I+\frac{4}{3}\left(\frac{3}{2}+\beta\right) C_{2} e^{(k)}+\frac{2}{9}(3+2 \beta)\left((4 \beta-3) C_{2}^{2}+6 C_{3}\right) e^{(k)^{2}} \\
& +\frac{4}{27}(2 \beta+3)\left(\left(8 \beta^{2}-12 \beta\right) C_{2}^{3}+(24 \beta+9) C_{2} C_{3}-18 C_{3} C_{2}+13 C_{4}\right) e^{(k)^{3}} \\
& +\frac{2}{81}(2 \beta+3)\left(144 \beta C_{3}^{2}+100 C_{5}\right) e^{(k)^{4}}+O\left(e^{(k)^{5}}\right)
\end{aligned}
$$


Then,

$$
\left(I-\frac{3}{4} A^{-1} B\right)\left[F^{\prime}\left(x^{(k)}\right)\right]^{-1} F\left(x^{(k)}\right)=e^{(k)}+\left(\left(1-\frac{8}{3} \beta\right) C_{2}^{3}+C_{3} C_{2}-\frac{1}{9} C_{4}\right) e^{(k)^{4}}+O\left(e^{(k)^{5}}\right) .
$$

Hence, the error equation is

$$
e^{(k+1)}=\left(\left(1-\frac{8}{3} \beta\right) C_{2}^{3}-C_{3} C_{2}+\frac{1}{9} C_{4}\right) e^{(k)^{4}}+O\left(e^{(k)^{5}}\right)
$$

and the proof is finished.

As this class of methods holds an infinity of specific procedures, it is necessary to extract some particular cases. The criterium to do this must be of efficiency, in some sense:

- When $\beta=-\frac{3}{2}$, the iterative expression becomes simpler,

$$
x^{(k+1)}=x^{(k)}-\left(I-\frac{3}{4}\left(u^{(k)}-\frac{3}{2} u^{(k)^{2}}\right)\right)\left[F^{\prime}\left(x^{(k)}\right)\right]^{-1} F\left(x^{(k)}\right) .
$$

- When $\beta=0$, classical Jarratt's method is found,

$$
x^{(k+1)}=x^{(k)}-\left(I-\frac{3}{4}[A]^{-1} u^{(k)}\right)\left[F^{\prime}\left(x^{(k)}\right)\right]^{-1} F\left(x^{(k)}\right),
$$

where $A=I+\frac{3}{2} u^{(k)}$.

- If $\beta=\frac{3}{8}$, the error equation is simpler,

$$
x^{(k+1)}=x^{(k)}-\left(I-\frac{3}{4}[A]^{-1} B\right)\left[F^{\prime}\left(x^{(k)}\right)\right]^{-1} F\left(x^{(k)}\right),
$$

where $A=I+\frac{15}{8} u^{(k)}$ and $B=A u^{(k)}=u^{(k)}+\frac{15}{8} u^{(k)^{2}}$.

These elements of the family can be, or not, the best ones. In order to have objective criteria to select them, we introduce in the following section a dynamical analysis of the family. This study will allow us to find the most stable and efficient elements of the class.

\section{Dynamical study of the family}

Firstly, some dynamical concepts of complex dynamics that are used in this work are shown (see [26]). Given a rational function $R: \hat{\mathbb{C}} \rightarrow \hat{\mathbb{C}}$, where $\hat{\mathbb{C}}$ is the Riemann sphere, the orbit of a point $z_{0} \in \hat{\mathbb{C}}$ is defined as

$$
\left\{z_{0}, R\left(z_{0}\right), R^{2}\left(z_{0}\right), \ldots, R^{n}\left(z_{0}\right), \ldots\right\} .
$$

A point $z_{0} \in \hat{\mathbb{C}}$, is called a fixed point of $R(z)$ if it verifies that $R(z)=z$. Moreover, $z_{0}$ is called a periodic point of period $p>1$ if it is a point such that $R^{p}\left(z_{0}\right)=z_{0}$ but $R^{k}\left(z_{0}\right) \neq z_{0}$, for each $k<p$. Moreover, a point $z_{0}$ is called pre-periodic if it is not periodic but there exists a $k>0$ such that $R^{k}\left(z_{0}\right)$ is periodic.

There exist different types of fixed points depending on its associated multiplier $\left|R^{\prime}\left(z_{0}\right)\right|$. Taking the associated multiplier into account, a fixed point $z_{0}$ is called:

- superattractor if $\left|R^{\prime}\left(z_{0}\right)\right|=0$,

- attractor if $\left|R^{\prime}\left(z_{0}\right)\right|<1$,

- repulsor if $\left|R^{\prime}\left(z_{0}\right)\right|>1$,

- and parabolic if $\left|R^{\prime}\left(z_{0}\right)\right|=1$. 
The fixed point operator of any iterative method on an arbitrary polynomial $p(z)$ is a rational function. The fixed points of this rational function that do not correspond to the roots of the polynomial $p(z)$ are called strange fixed points. On the other hand, a critical point $z_{0}$ is a point which satisfies that $R^{\prime}\left(z_{0}\right)=0$.

The basin of attraction of an attractor $\alpha$ is defined as

$$
\mathcal{A}(\alpha)=\left\{z_{0} \in \hat{\mathbb{C}}: R^{n}\left(z_{0}\right) \rightarrow \alpha, n \rightarrow \infty\right\}
$$

The Fatou set of the rational function $R, \mathcal{F}(R)$, is the set of points $z \in \hat{\mathbb{C}}$ whose orbits tend to an attractor (fixed point, periodic orbit or infinity). Its complement in $\hat{\mathbb{C}}$ is the Julia set, $\mathcal{J}(R)$. That means that the basin of attraction of any fixed point belongs to the Fatou set and the boundaries of these basins of attraction belong to the Julia set.

The fixed point operator associated to the family of methods (2) in the scalar case, on a nonlinear function $f(z)$ is

$$
G(z)=z-\left(1-\frac{3}{4} \frac{u(1+\beta u)}{1+u\left(\beta+\frac{3}{2}\right)}\right) \frac{f(z)}{f^{\prime}(z)},
$$

where $u=\frac{f^{\prime}(y)-f^{\prime}(z)}{f^{\prime}(z)}$ and $y$ is

$$
y=z-\frac{2}{3} \frac{f(z)}{f^{\prime}(z)}
$$

By applying this operator on a generic polynomial $p(z)=(z-a)(z-b)$, and by using the Möebius map $h(z)=\frac{z-a}{z-b}$, whose properties are

$$
\text { i) } h(\infty)=1, \quad \text { ii) } h(a)=0, \quad \text { iii) } h(b)=\infty \text {, }
$$

the rational operator associated to the family of iterative schemes is finally

$$
O p(z, \beta)=\frac{z^{4}\left(8 \beta-3 z^{2}+(4 \beta-6) z-3\right)}{(8 \beta-3) z^{2}+(4 \beta-6) z-3} .
$$

\subsection{Study of the fixed points and their stability}

It is clear that $z=0$ and $z=\infty$ are fixed points of $O p(z, \beta)$ (related to the roots $a$ and $b$, respectively, of the polynomial $p(z))$. On the other hand, we can see, by solving the equation $O p(z, \beta)=z$, that $z=1$ is a strange fixed point, which is associated with the original convergence to infinity. Moreover, there are also another four strange fixed points which correspond with the roots of the polynomial

$$
q(z)=3+(9-4 \beta) z+12(1-\beta) z^{2}+(9-4 \beta) z^{3}+3 z^{4}
$$

whose analytical expressions, depending on $\beta$, are:

$$
\begin{aligned}
& e x_{1}(\beta)=\frac{1}{12}\left(-9+4 \beta-\sqrt{9+8 \beta(9+2 \beta)}-\sqrt{32 \beta^{2}-8 \beta \sqrt{9+8 \beta(9+2 \beta)}+18(-3+\sqrt{9+8 \beta(9+2 \beta)})}\right) \\
& e x_{2}(\beta)=\frac{1}{12}\left(-9+4 \beta-\sqrt{9+8 \beta(9+2 \beta)}+\sqrt{32 \beta^{2}-8 \beta \sqrt{9+8 \beta(9+2 \beta)}+18(-3+\sqrt{9+8 \beta(9+2 \beta)})}\right) \\
& e x_{3}(\beta)=\frac{1}{12}(-9+4 \beta+\sqrt{9+8 \beta(9+2 \beta)}-\sqrt{-18(3+\sqrt{9+8 \beta(9+2 \beta)})+8 \beta(4 \beta+\sqrt{9+8 \beta(9+2 \beta)})}) \\
& \operatorname{ex}_{4}(\beta)=\frac{1}{12}(-9+4 \beta+\sqrt{9+8 \beta(9+2 \beta)}+\sqrt{-18(3+\sqrt{9+8 \beta(9+2 \beta)})+8 \beta(4 \beta+\sqrt{9+8 \beta(9+2 \beta)})})
\end{aligned}
$$

There exist relations between the strange fixed points and they are described in the following result.

Lemma 1 The number of simple strange fixed points of operator $O p(z, \beta)$ is five, except in the following cases: 
i) If $\beta=0$, then $\operatorname{ex}_{1}(\beta)=\mathrm{ex}_{2}(\beta)=-1$ that is not a fixed point, so there only exist three simple strange fixed points.

ii) If $\beta=\frac{9}{5}$, then $\operatorname{ex}_{3}(\beta)=e x_{4}(\beta)=1$, and as a consequence the family has three strange fixed points, one of them of multiplicity three.

iii) If $\beta=\frac{3}{4}(-3-2 \sqrt{2})$ or $\beta=\frac{3}{4}(-3+2 \sqrt{2})$, then $\operatorname{ex}_{1}(\beta)=e x_{3}(\beta)$ and $e x_{2}(\beta)=e x_{4}(\beta)$ and there exist three strange fixed points, two of them double.

Moreover, for all values of the parameter $\beta$, $\operatorname{ex}_{1}(\beta)=\frac{1}{e x_{2}(\beta)}$ and $\operatorname{ex}_{3}(\beta)=\frac{1}{e x_{4}(\beta)}$.

Related to the stability of that strange fixed points, the first derivative of $O p(z, \beta)$ must be calculated

$$
O p^{\prime}(z, \beta)=\frac{12 z^{3}(1+z)^{2}\left(3+6 z+3 z^{2}-8 \beta-4 z \beta-8 z^{2} \beta+8 z \beta^{2}\right)}{\left(-3-6 z-3 z^{2}+4 z \beta+8 z^{2} \beta\right)^{2}} .
$$

Taking into account that the methods of family (2) have order of convergence four and the origin and $\infty$ correspond to the roots of $p(z)$ from Möebius map, it is immediate that the origin and $\infty$ are superattractive fixed points for every value of $\beta$.

The stability of the other fixed points is more complicated and will be shown in a separate way. First of all, focussing the attention in the strange fixed point $z=1$, which is related to the original convergence to $\infty$, and the following result can be shown.

Due to the complexity of the stability function of each one of the strange fixed points, $O p^{\prime}\left(e x_{i}, \beta\right)$, to characterize its domain analytically is not affordable. We will use the graphical tools of software Mathematica in order to obtain the regions of stability of each of them (those $z \in \mathbb{C}$ such that $\left|O p^{\prime}(z, \beta)\right|<1$ ), in the complex plane. In Figure 1, the stability region of $z=1$ can be observed, whose shape is an oval in the complex plane, symmetric respect to the real axis. In Figure 2, the regions of stability of $e x_{1}(\beta)$ and $e x_{3}(\beta)$ are shown (the ones of $e x_{2}(\beta)$ and $e x_{4}(\beta)$ are the same respectively, as the points are conjugated). Taking into account these regions the following result summarize the behavior of the strange fixed points.

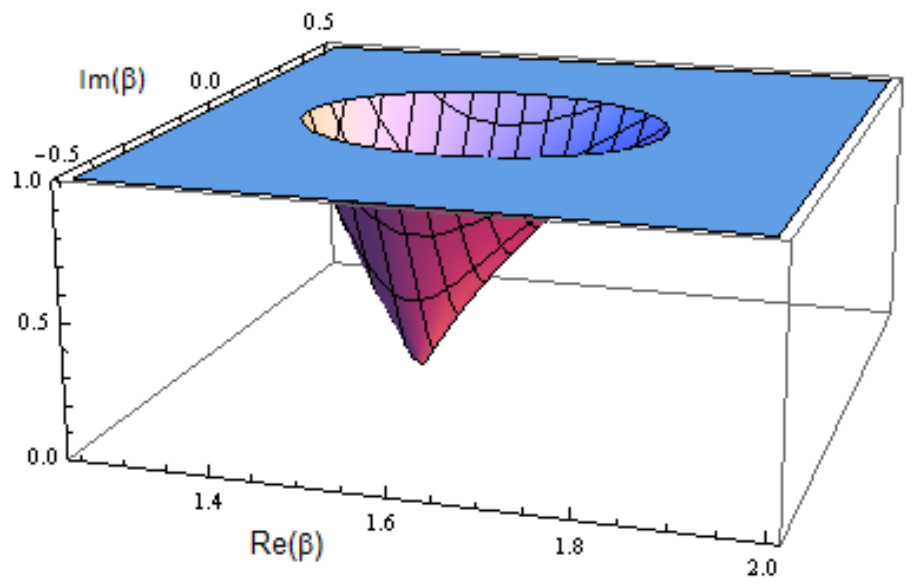

Figure 1: Stability region of $z=1$

Theorem 2 The strange fixed point $z=1$ is super-attractor if $\beta=1.5$ or if $\beta$ is inside the oval that appears in Figure 1. On the other hand:

- The stability of $e_{1}(\beta)$ and $e x_{2}(\beta)$ is the same. In particular, they are attractive if $\beta$ is inside the cardioid that appears in the left hand of Figure 2.

- The stability of $\operatorname{ex}_{3}(\beta)$ and $e x_{4}(\beta)$ is the same. In particular, they are attractive if $\beta$ is inside the oval that appears in the right hand of Figure 2.

As a conclusion we can remark that the number and the stability of the fixed points depend on the parameter $\beta$. 

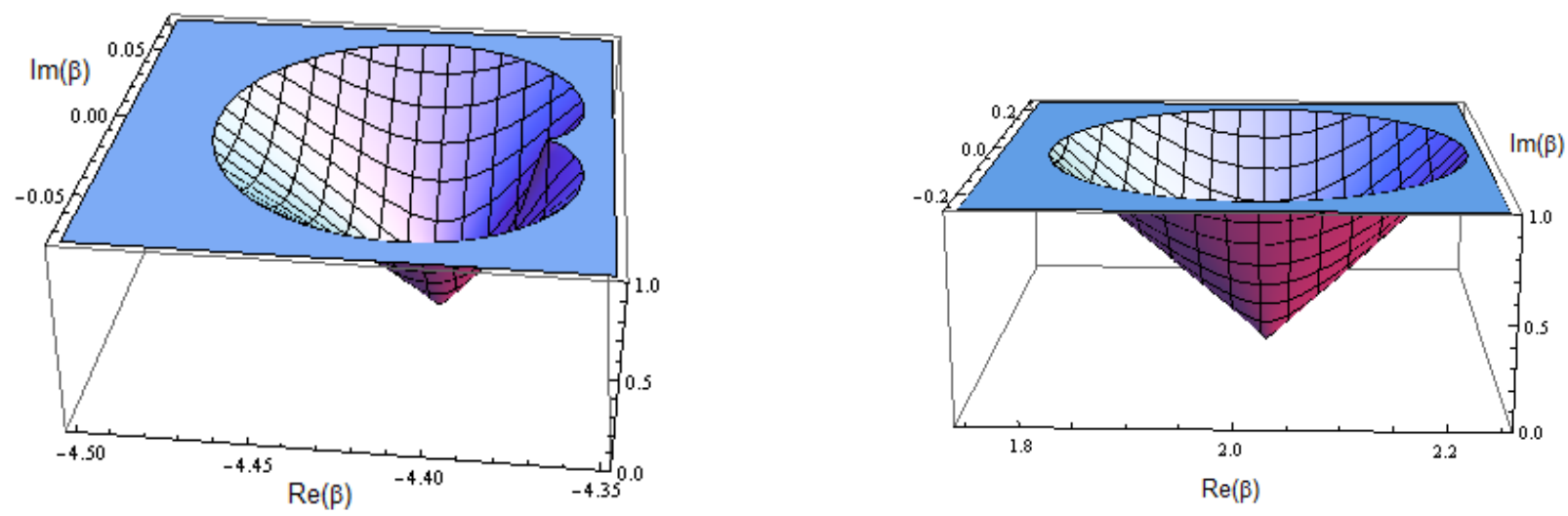

Figure 2: Stability region of $e x_{1}(\beta)$ and $e x_{3}(\beta)$

\subsection{Study of the critical points and parameter spaces}

In this section, the critical points will be calculated and the parameter spaces associated to the free critical points will be shown. It is well known that there is at least one critical point associated with each invariant Fatou component. The critical points of the family are the solutions of $O p^{\prime}(z, \beta)=0$, where $O p^{\prime}(z, \beta)$ is described in (8).

By solving this equation, it is clear that $z=0$ and $z=\infty$ are critical points, which are related to the roots of the polynomial $p(z)$ and they have associated their own Fatou component. Moreover, there exist critical points no related to the roots, these points are called free critical points. Their expressions are:

$$
\begin{gathered}
c r_{0}=-1, \\
c r_{1}(\beta)=\frac{3-2 \beta+4 \beta^{2}-2 \sqrt{9 \beta-9 \beta^{2}-4 \beta^{3}+4 \beta^{4}}}{-3+8 \beta}
\end{gathered}
$$

and

$$
c r_{2}(\beta)=\frac{3-2 \beta+4 \beta^{2}+2 \sqrt{9 \beta-9 \beta^{2}-4 \beta^{3}+4 \beta^{4}}}{-3+8 \beta} .
$$

The relations between the free critical points are described in the following result.

Lemma 2 a) If $\beta=0$ or $\beta=-3 / 2$

(i) $c r_{1}=c r_{2}=-1$.

b) If $\beta=1$ or $\beta=3 / 2$

(i) $c r_{1}=c r_{2}=1$.

c) For other values of $\beta$

(i) The family has 3 free critical points.

It is easy to see that $z=-1$ is a pre-periodic point as it is the pre-image of the fixed point related to the convergence to infinity, $z=1$, and the other free critical points are conjugated $c r_{1}(\beta)=1 / c r_{2}(\beta)$. So, there are only two independent free critical points and only one is not pre-periodic. Without loss of generality, we consider in this paper the free critical point $c r_{1}(\beta)$. In order to find the best members of the family in terms of stability, the parameter space corresponding to this independent free critical point will be shown.

The study of the orbits of the critical points gives rise about the dynamical behavior of an iterative method. In concrete, to determine if there exists any attracting strange fixed point or periodic orbit, the following question must be answered: For which values of the parameter, the orbits of the free critical points are attracting periodic orbits? In order to answer this question we are going to draw the parameter space [27]. In it, each point is 


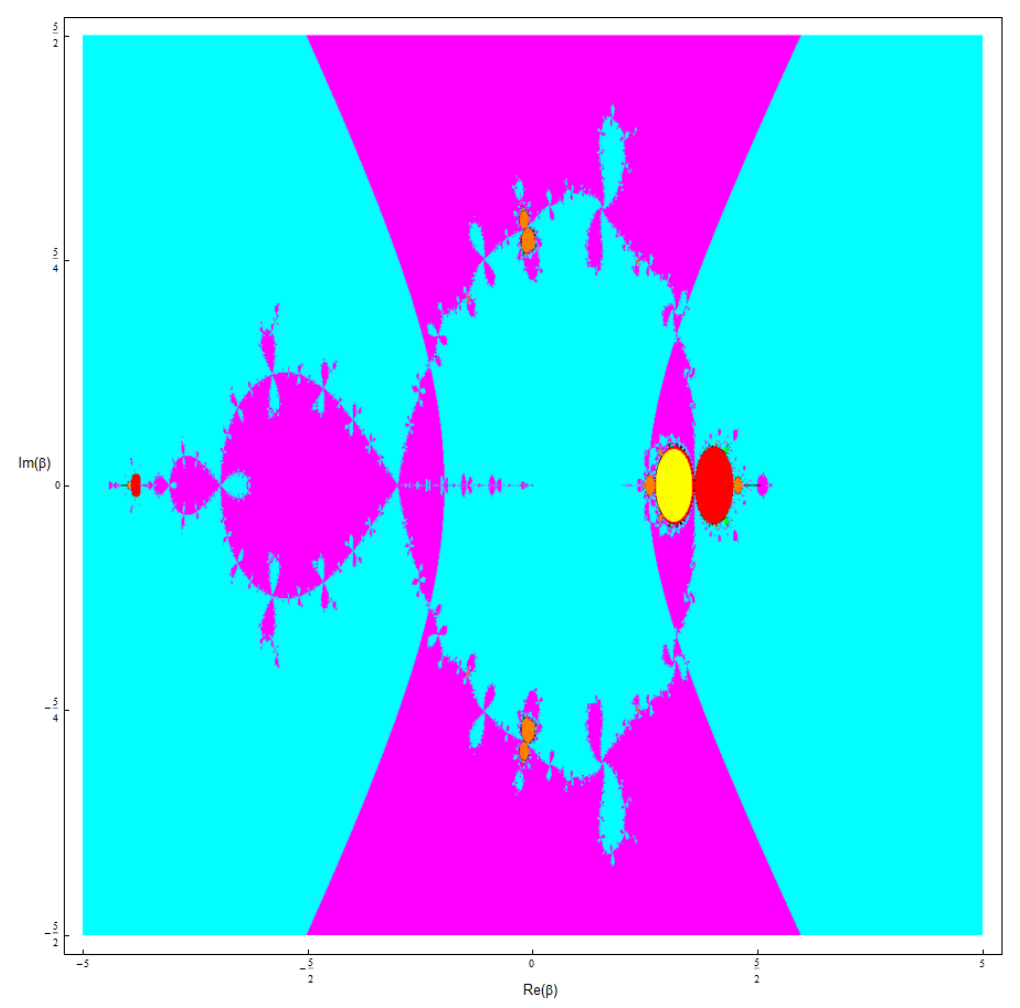

Figure 3: Parameter space associated to the free critical point $\mathrm{cr}_{1}(\beta)$.

associated to a complex value of parameter $\beta$, that is, to an element of the family of iterative methods. When the critical point is used as an initial estimation, for each value of the parameter, the color of the point tell us about the place it has converged to: to a fixed point, to an attracting periodic orbit or even the infinity.

In Figure 3, the parameter space associated to $\mathrm{cr}_{1}(\beta)$ is shown. A point is painted in cyan if the iteration of the method starting in $z_{0}=c r_{1}(\beta)$ converges to the fixed point 0 (related to root $a$ ), in magenta if it converges to $\infty$ (related to root $b$ ) and in yellow if the iteration converges to 1 (related to $\infty$ ). Moreover, it appears in red the convergence, after a maximum of 2000 iterations and with a tolerance of $10^{-6}$, to any of the strange fixed points, in orange the convergence to 2-cycles, in light green the convergence to 3-cycles, in dark red to 4-cycles, in dark blue to 5-cycles, in dark green to 6-cycles, dark yellow to 7-cycles, and in white the convergence to 8-cycles. The regions in black correspond to zones of convergence to other cycles. As a consequence, every point of the plane which is neither cyan nor magenta is not a good choice of $\beta$ in terms of numerical behavior.

In Figure 4 some details of the parameter spaces are shown. It can be seen that in this region there exist values of $\beta$ for which the iteration of $\operatorname{cr}_{1}(\beta)$ converges to cycles, strange fixed points or even to $z=1$. In concrete, in the left hand it can be observed two large circular zones: the yellow one corresponds to values of $\beta$ for which $z=1$ is (super)attractive and the red one is the region where the iteration of the free critical point converges to some of the other strange fixed points.

Once the values of the parameter where anomalies appear have been detected, the next step consists on finding them in the dynamical planes. In these dynamical planes the convergence to 0 appear in magenta, in cyan it appears the convergence to $\infty$ and in black the zones with no convergence to the roots. First of all, in Figure 5 and Figure 6 the dynamical planes associated with the values of $\beta$ for which there is no convergence problems, are shown.

Then, focussing the attention in the region shown in Figure 4 it is evident that there exist members of the family with complicated behavior. In Figure 7, the dynamical planes of some of this members are shown. In concrete, in the left hand appears the dynamical plane of a member of the family with regions of convergence to any of the strange fixed points, and in the right hand the dynamical plane of a member of the family with regions of convergence to $z=1$, which is related to the convergence to $\infty$, is shown.

Finally, in Figure 8 and Figure 9, some dynamical planes of members of the family with convergence to different attracting cycles are shown. 

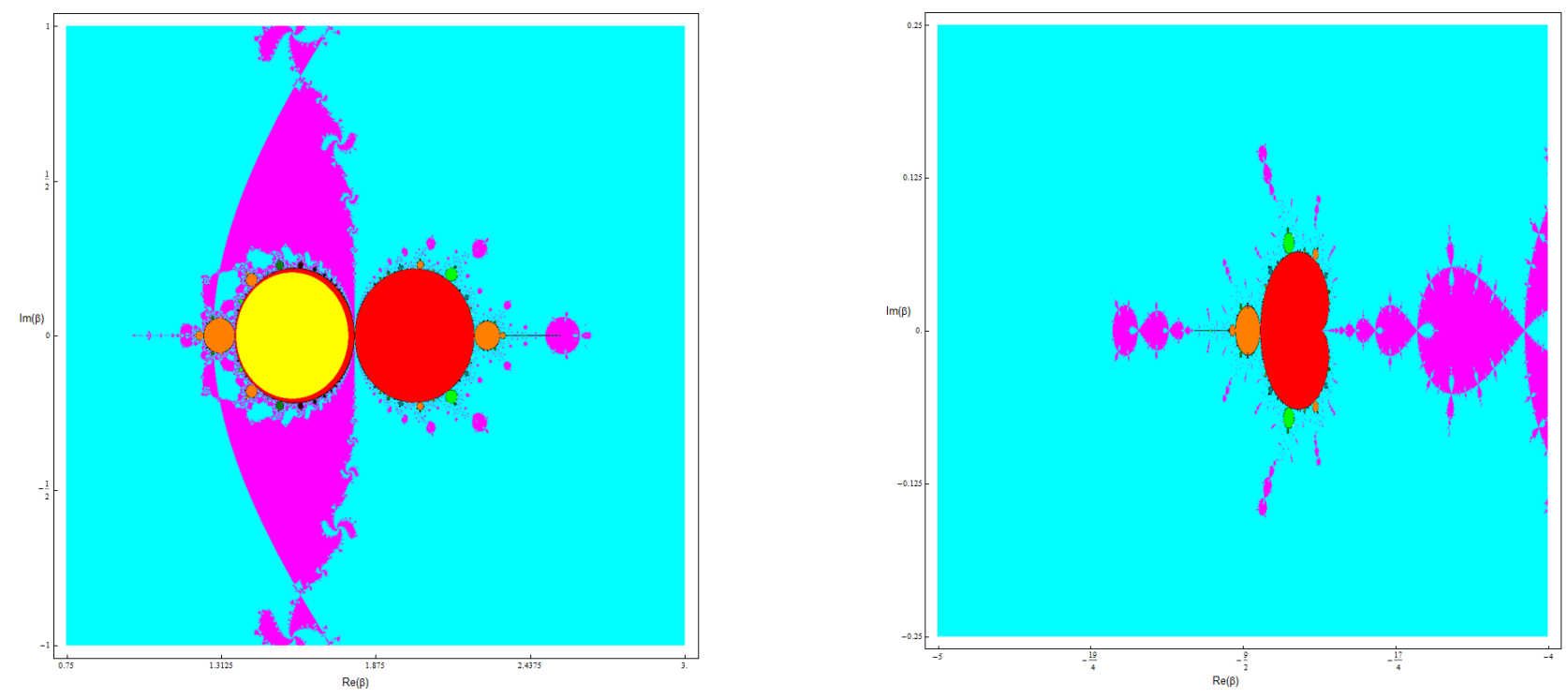

Figure 4: Details of parameter space associated to the free critical point $c r_{1}(\beta)$, where there exist regions with convergence problems.
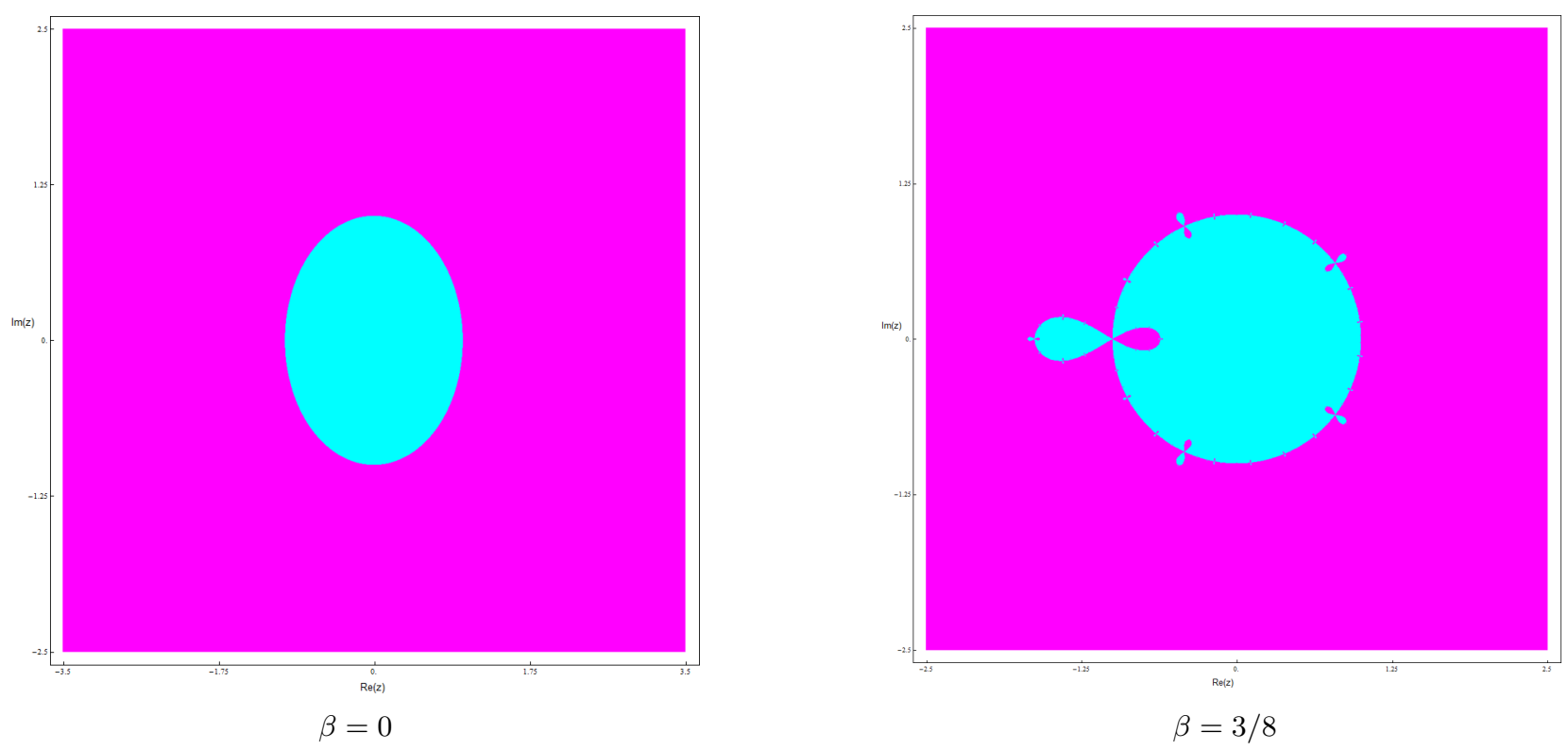

Figure 5: Dynamical planes for different values of $\beta$ in which there are no convergence anomalies.

\section{Numerical results}

To solve the equation of molecular interaction, (see [28])

$$
\begin{array}{rc}
u_{x x}+u_{y y}=u^{2}, & (x, y) \in[0,1] \times[0,1] \\
u(x, 0)=2 x^{2}-x+1, & u(x, 1)=2 \\
u(0, y)=2 y^{2}-y+1, & u(1, y)=2
\end{array}
$$

we need to deal with a boundary value problem with a nonlinear partial differential equation of second order. To estimate its solution numerically, we have used central divided differences in order to transform the problem in a nonlinear system of equations, which is solved by using some elements of the proposed family (for specific 

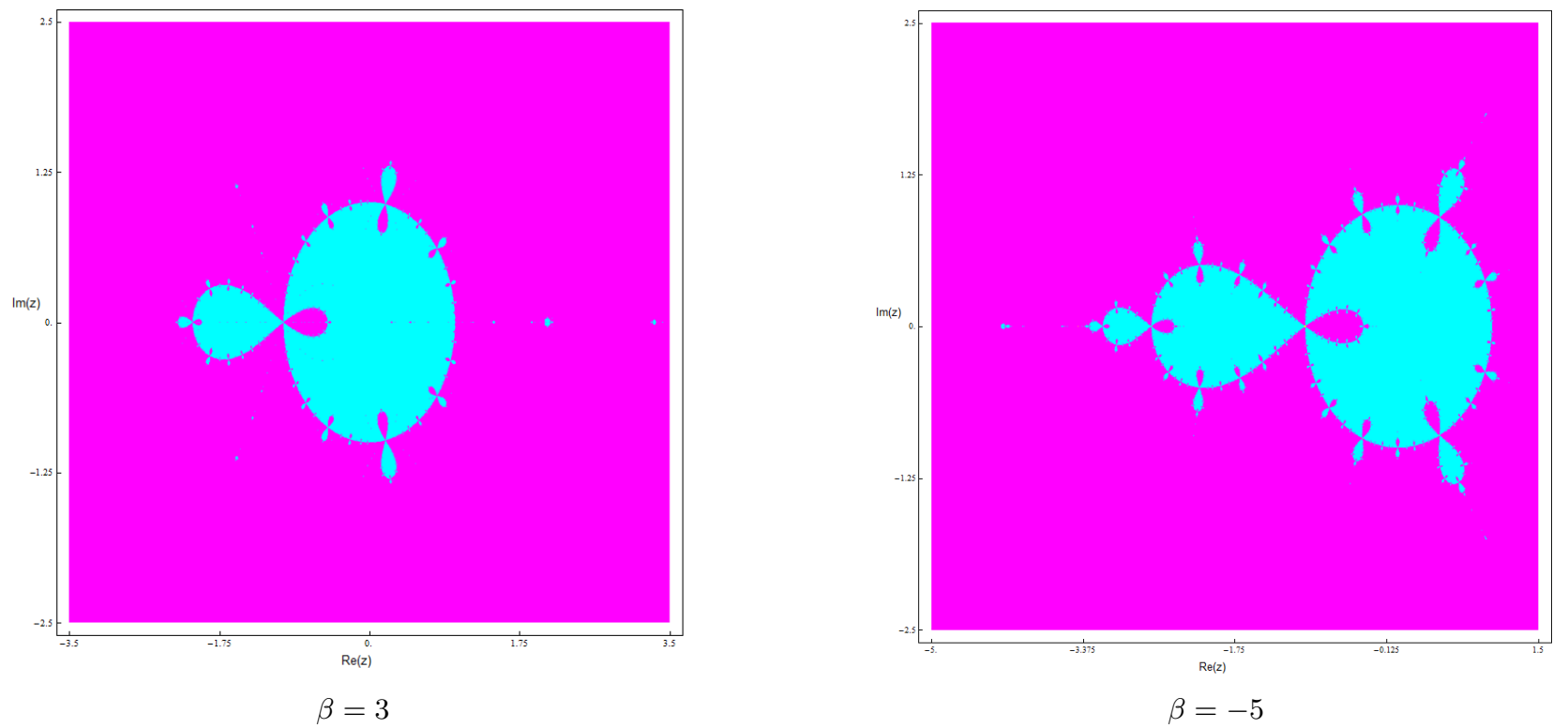

Figure 6: Dynamical planes for different values of $\beta$ in which there are no convergence anomalies.
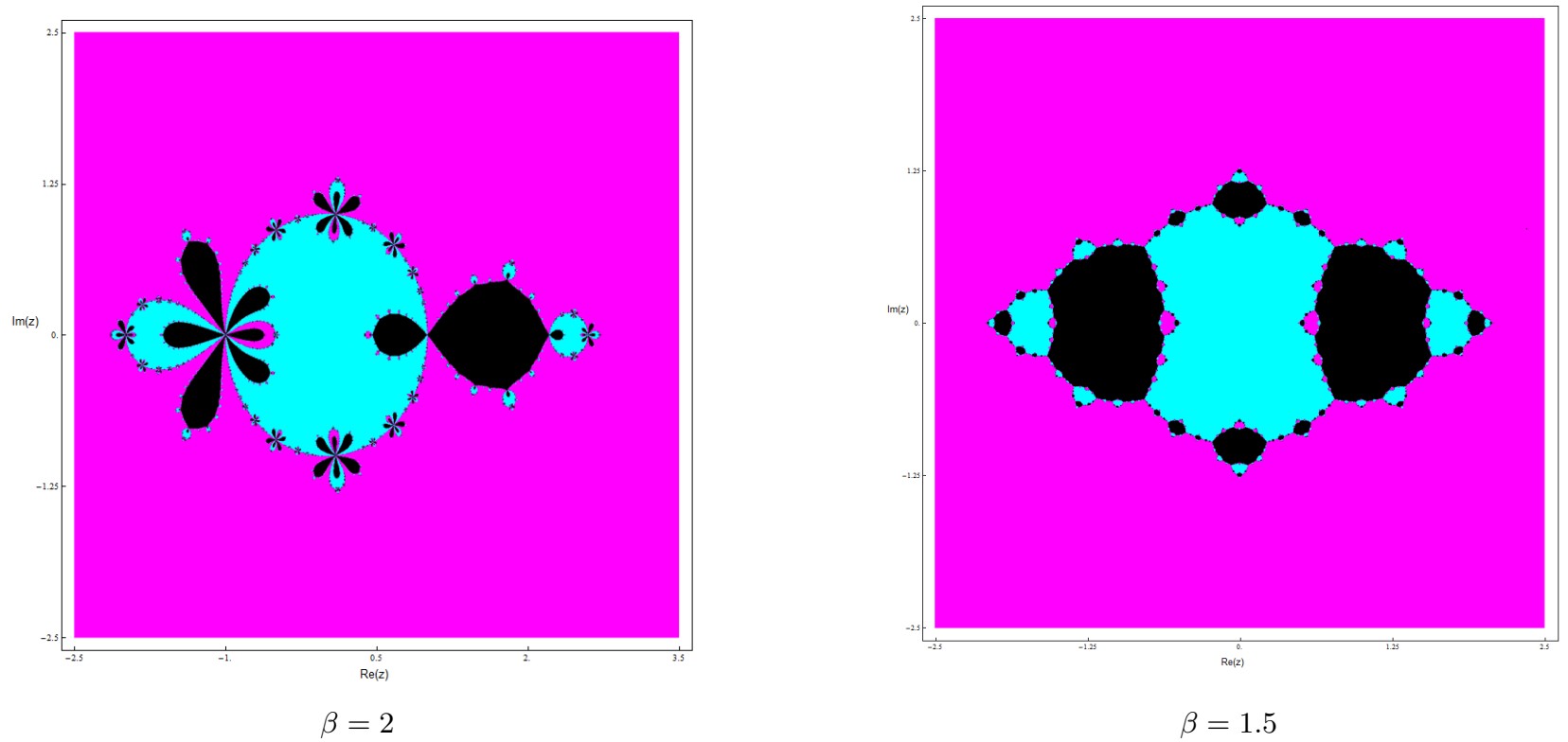

Figure 7: Dynamical planes for different values of $\beta$.

values of the parameter $\beta$ ) and also by known methods, as Newton' and Sharma's ones ([29]), which has order four and its iterative expression is

$$
\begin{aligned}
y^{(k)} & =x^{(k)}-\frac{2}{3}\left[F^{\prime}\left(x^{(k)}\right)\right]^{-1} F\left(x^{(k)}\right), \\
x^{(k+1)} & =x^{(k)}-\frac{1}{2}\left[-I+\frac{9}{4}\left[F^{\prime}\left(y^{(k)}\right)\right]^{-1} F^{\prime}\left(x^{(k)}\right)+\frac{3}{4}\left[F^{\prime}\left(x^{(k)}\right)\right]^{-1} F^{\prime}\left(y^{(k)}\right)\right]\left[F^{\prime}\left(x^{(k)}\right)\right]^{-1} F\left(x^{(k)}\right) .
\end{aligned}
$$

The discretization process yields to the nonlinear system of equations,

$$
u_{i+1, j}-4 u_{i, j}+u_{i-1, j}+u_{i, j+1}+u_{i, j-1}-h^{2} u_{i, j}^{2}=0 \quad i=1, \ldots, n x, \quad j=1, \ldots, n y,
$$



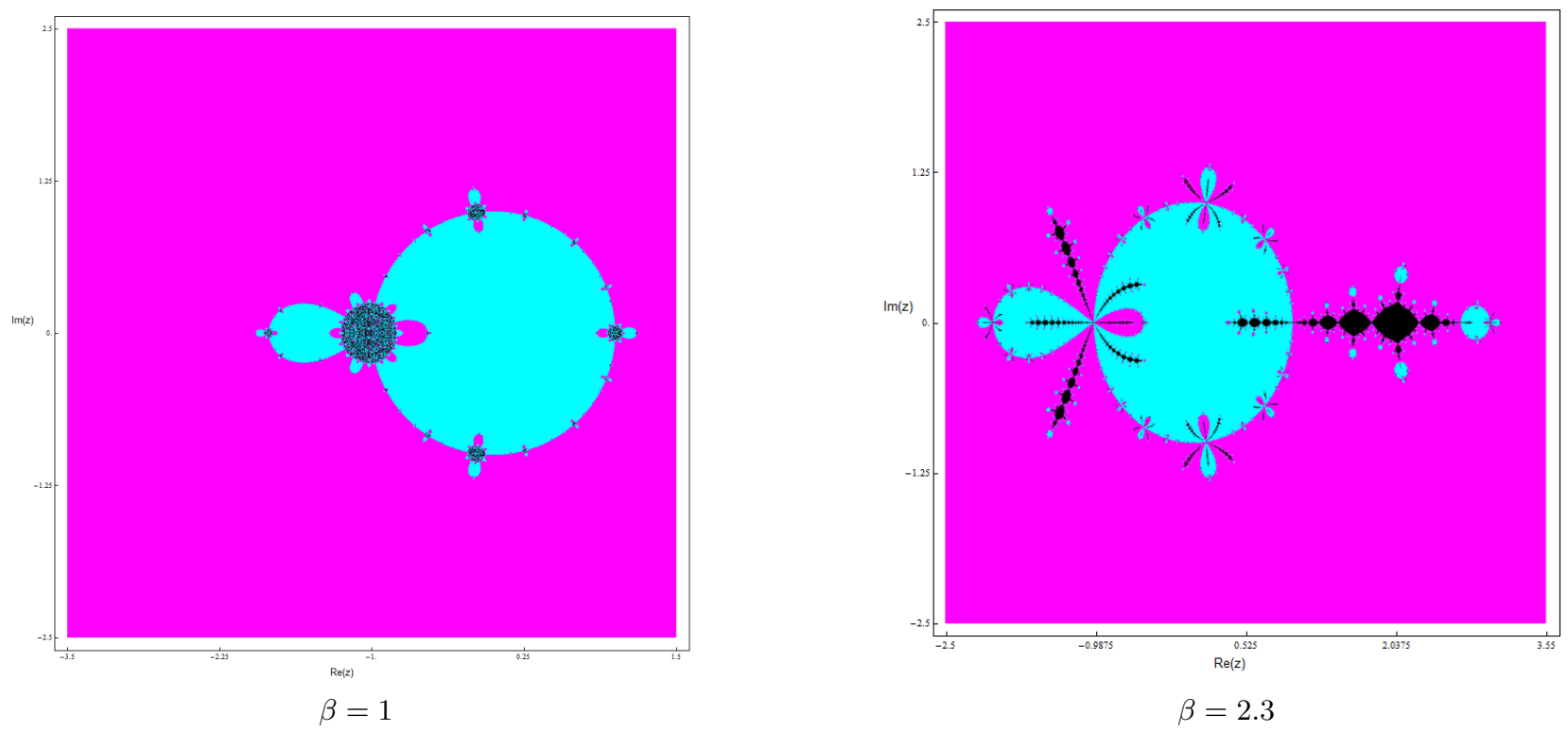

Figure 8: Dynamical planes for different values of $\beta$ for which there exist attracting cycles
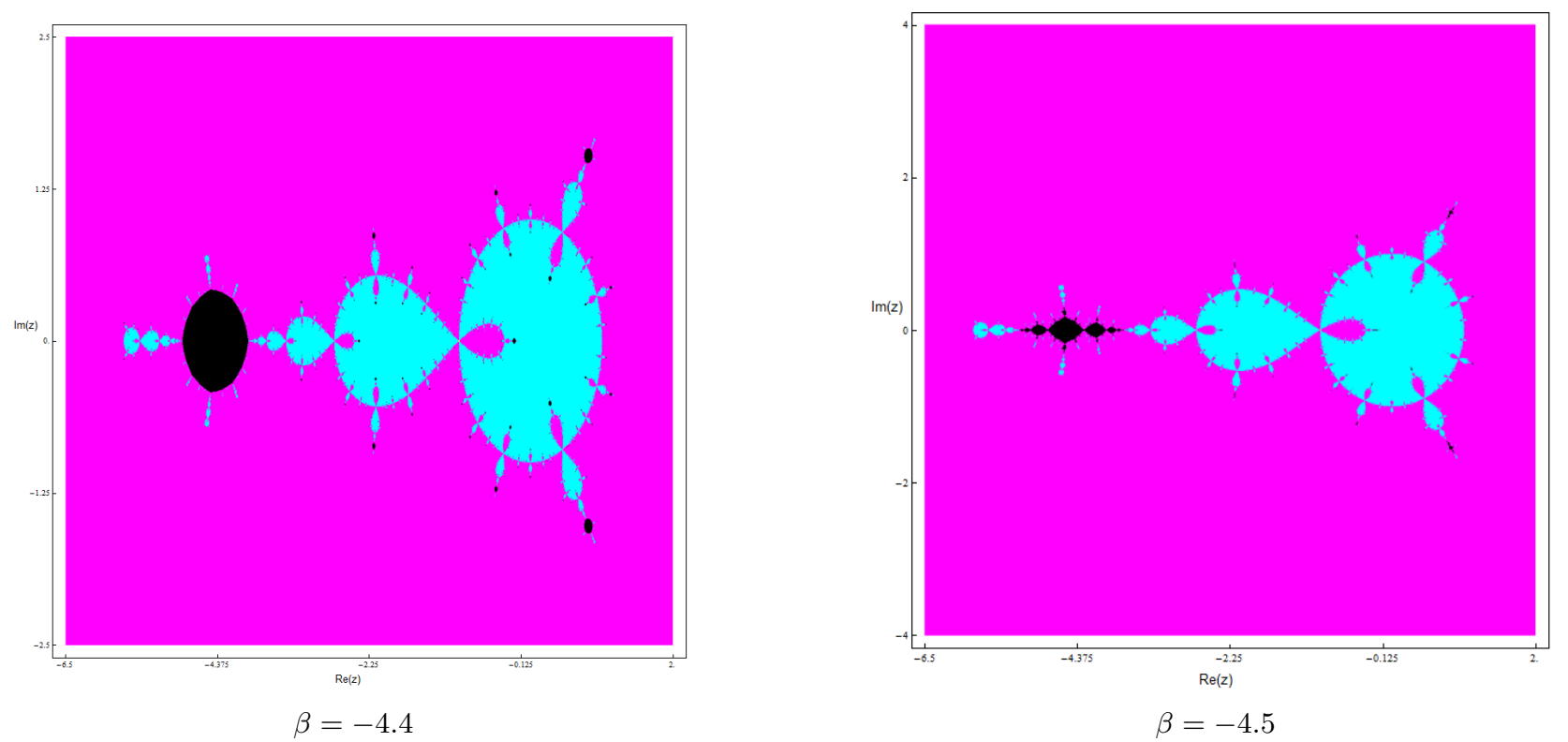

Figure 9: Dynamical planes for different values of $\beta$ for which there exist attracting cycles

where $u_{i, j}$ denotes the estimation of the unknown $u\left(x_{i}, y_{j}\right), x_{i}=i h$ with $i=0,1, \ldots, n x, y_{j}=j k$ with $j=0,1, \ldots, n y$, are the nodes in both variables, being $h=k=\frac{1}{n x}=\frac{1}{n y}$.

In this case, we fix $n x=n y=4$, so a mesh of $5 \times 5$ is generated. As the boundary conditions give us the value of the unknown function at the nodes $\left(x_{0}, y_{j}\right),\left(x_{4}, y_{j}\right)$ for all $j$ and also at $\left(x_{i}, y_{0}\right),\left(x_{i}, y_{4}\right)$ for all $i$, we have only nine unknowns, that are renamed as:

$$
x_{1}=u_{1,1}, \quad x_{2}=u_{2,1}, \quad x_{3}=u_{3,1}, x_{4}=u_{1,2}, \quad x_{5}=u_{2,2}, \quad x_{6}=u_{3,2}, x_{7}=u_{1,3}, \quad x_{8}=u_{2,3}, \quad x_{9}=u_{3,3} .
$$

So, the system can be expressed as

$$
F(x)=A x+\phi(x)-b=0, \quad F^{\prime}(x)=A+2 h^{2} \operatorname{diag}\left(x_{1}, x_{2}, \ldots, x_{9}\right)
$$


where

$$
A=\left(\begin{array}{ccc}
M & -I & 0 \\
-I & M & -I \\
0 & -I & M
\end{array}\right), \quad \text { being } \quad M=\left(\begin{array}{ccc}
4 & -1 & 0 \\
-1 & 4 & -1 \\
0 & -1 & 4
\end{array}\right), \quad \phi(x)=h^{2}\left(x_{1}^{2}, x_{2}^{2}, \ldots, x_{9}^{2}\right)^{T}
$$

$I$ is the $3 \times 3$ identity matrix and $b=\left(\frac{7}{4}, 1, \frac{27}{8}, 1,0,2, \frac{27}{8}, 2,4\right)^{T}$. Now, we will check the performance of the methods by means of some numerical tests, by using variable precision arithmetics of 2000 digits of mantissa. These tests have been made by using the stopping criterium $\left\|F\left(x^{(k+1)}\right)\right\|+\left\|x^{(k+1)}-x^{(k)}\right\|<10^{-500}$. In this way we assure that, in case of convergence, we have converged to a solution. In Table 1, we show the numerical results obtained for the problem of molecular interaction (10), with different initial estimations. We show, for each method, the number of iterations, the residual of the function at the last iteration, $\left\|F\left(x^{(k+1)}\right)\right\|$ and the approximated computational order of convergence $A C O C$ defined in the Introduction. The value of $A C O C$ that appears in Table 1 is the last coordinate of vector $A C O C$ when the variation between its values is small.

\begin{tabular}{|c|c|c|c|c|}
\hline & & $x^{(0)}=(1, \ldots, 1)$ & $x^{(0)}=(5, \ldots, 5)$ & $x^{(0)}=25(1,-1, \ldots,-1,1)$ \\
\hline \multirow{6}{*}{ iter } & Newton & 9 & 11 & $<10^{3}$ \\
\hline & Sharma & 5 & 6 & $<10^{3}$ \\
\hline & Jarratt & 5 & 6 & $<10^{3}$ \\
\hline & $\beta=\frac{3}{8}$ & 5 & 6 & 10 \\
\hline & $\beta=-\frac{1}{6}$ & 5 & 6 & $<10^{3}$ \\
\hline & $\beta=-1$ & 5 & 6 & 176 \\
\hline \multirow{6}{*}{$\mathrm{ACOC}$} & Newton & 1.9999 & 2.0000 & - \\
\hline & Sharma & 3.9962 & 3.9981 & - \\
\hline & Jarratt & 3.9964 & 3.9988 & - \\
\hline & $\beta=\frac{3}{8}$ & 4.0519 & 4.0184 & 4.0402 \\
\hline & $\beta=-\frac{1}{6}$ & 3.9963 & 3.9986 & - \\
\hline & $\beta=-1$ & 3.9960 & 3.9976 & - \\
\hline \multirow{6}{*}{$\left\|F\left(x^{(k)}\right)\right\|$} & Newton & $6.45 \mathrm{e}-828$ & $2.06 \mathrm{e}-1161$ & - \\
\hline & Sharma & $1.56 \mathrm{e}-1531$ & $7.84 \mathrm{e}-1899$ & - \\
\hline & Jarratt & $5.05 \mathrm{e}-1657$ & $1.74 \mathrm{e}-2007$ & - \\
\hline & $\beta=\frac{3}{8}$ & $1.48 \mathrm{e}-2007$ & $2.61 \mathrm{e}-2007$ & $2.78 \mathrm{e}-2007$ \\
\hline & $\beta=-\frac{1}{6}$ & $6.42 \mathrm{e}-1603$ & $1.98 \mathrm{e}-2007$ & - \\
\hline & $\beta=-1$ & $2.6 \mathrm{e}-1463$ & $1.09 \mathrm{e}-1612$ & $3.64 \mathrm{e}-1562$ \\
\hline
\end{tabular}

Table 1: Numerical tests

In Table 1, we notice that the performance of the selected elements of the proposed family is at least equal and sometimes better than the classical known schemes. The elements of the class that showed good stability in the dynamical study (most of them around 0 , like $\beta= \pm \frac{1}{6}$ ) hold very good behavior. In fact, the best results are obtained for $\beta=\frac{3}{8}$, which simplifies the error equation and presents a basin of good starting points wider than the rest of methods, as we can observe in the last column of Table 1, where the initial estimation is very far from the solution $\alpha_{1}$ of the problem, which is approximately shown in Table 2. Although all the methods converge to $\alpha_{1}$ for $x^{(0)}=(1, \ldots, 1)$ and $x^{(0)}=(5, \ldots, 5)$, a second solution $\alpha_{2}$ of the nonlinear system appears when $x^{(0)}=(25,-25, \ldots,-25,25)$ for $\beta=-1$.

\section{Conclusions}

A family of Jarratt-type iterative root-finding algorithms has been extensively studied in terms of stability and convergence. All the fixed points has been calculated and their stability has been also analyzed. Moreover, by means of the parameter space, some anomalies such us convergence to the strange fixed points, convergence to cycles or even chaotical behavior has been found. Some dynamical planes are presented validating the results given. The main conclusion drawn from this study is that the stability of a member of the family depends clearly on the value of the parameter that defines it. By applying stable selected elements of this family, we obtain good numerical results solving the equation of molecular interaction. 


\begin{tabular}{|c|c|c|}
\hline \hline & $\alpha_{1}$ & $\alpha_{2}$ \\
\hline$u_{1,1}$ & $1.0259117 \ldots$ & $-9.2167742 \ldots$ \\
$u_{2,1}$ & $1.2097139 \ldots$ & $-16.653894 \ldots$ \\
$u_{3,1}$ & $1.5167030 \ldots$ & $-8.2014229 \ldots$ \\
$u_{1,2}$ & $1.2097139 \ldots$ & $-16.653894 \ldots$ \\
$u_{2,2}$ & $1.3877038 \ldots$ & $-32.862868 \ldots$ \\
$u_{3,2}$ & $1.6258725 \ldots$ & $-15.322839 \ldots$ \\
$u_{1,3}$ & $1.5167030 \ldots$ & $-8.2014229 \ldots$ \\
$u_{2,3}$ & $1.6258725 \ldots$ & $-15.322839 \ldots$ \\
$u_{3,3}$ & $1.7642995 \ldots$ & $-7.5527268 \ldots$ \\
\hline \hline
\end{tabular}

Table 2: Approximated solutions

Acknowledgments: The authors thank to the anonymous referees for their suggestions to improve the paper.

\section{References}

[1] R.C. Rach, J.S. Duan, A.M. Wazwaz, Solving coupled Lane-Emden boundary value problems in catalytic diffusion reactions by the Adomian decomposition method. Journal of Mathematical Chemistry 52(1), 255-267 (2014).

[2] R. Singh, G. Nelakanti, J. Kumar, A new efficient technique for solving two-point boundary value problems for integro-differential equations. Journal of Mathematical Chemistry doi: 10.1007/s10910-014-0363-8.

[3] M. Mahalakshmi, G. Hariharan, K. Kannan, The wavelet methods to linear and nonlineal reaction-diffusion model arising in mathematical chemistry. Journal of Mathematical Chemistry 51, 2361-2385 (2013).

[4] P.G. Logrado, J.D.M. Vianna, Partitioning technique procedure revisited: Formalism and first application to atomic problems. Journal of Mathematical Chemistry 22, 107-116 (1997).

[5] C.G. Jesudason, I. Numerical nonlinear analysis: differential methods and optimization applied to chemical reaction rate determination. Journal of Mathematical Chemistry 49, 1384-1415 (2011).

[6] A. Klamt, Conductor-like Screening Model for Real Solvents: A New Approach to the Quantitative Calculation of Solvation Phenomena, J. Phys. Chem., 99, 2224-2235 (1995).

[7] A. Klamt, V. Jonas, T. Brger, J. C. W. Lohrenz, Refinement and Parametrization of COSMORS, J. Phys. Chem. A, 102, 5074-5085 (1998).

[8] H. Grensemann, J. Gmehling, Performance of a Conductor-Like Screening Model for Real Solvents Model in Comparison to Classical Group Contribution Methods, Ind. Eng. Chem. Res., 44(5), 1610-1624 (2005).

[9] T. Banerjee, A. Khanna, Infinite Dilution Activity Coefficients for Trihexyltetradecyl Phosphonium Ionic Liquids: Measurements and COSMO-RS Prediction, J. Chem. Eng. Data, 51(6), 2170-2177 (2006).

[10] R. Franke, B. Hannebauer, On the influence of basis sets and quantum chemical methods on the prediction accuracy of COSMO-RS, Phys. Chem. Chem. Phys., 13, 21344-21350 (2011).

[11] K. Maleknejad, M. Alizadeh, An Efficient Numerical Scheme for Solving Hammerstein Integral Equation Arisen in Chemical Phenomenon, Procedia Computer Science, 3, 361-364 (2011).

[12] M. Petković, B. Neta, L. Petković, J. Džunić, Multipoint methods for solving nonlinear equations, Academic Press, 2012.

[13] A. Cordero, J.R. Torregrosa, Variants of Newton's Method using fifth-order quadrature formulas. Applied Mathematics and Computation 190, 686-698 (2007).

[14] H.T. Kung, J.F. Traub, Optimal order of one-point and multi-point iterations. J. Assoc. Comput. Math. 21, 643-651, (1974). 
[15] A.M. Ostrowski, Solution of equations and systems of equations, Prentice-Hall, Englewood Cliffs, NJ, USA, 1964.

[16] P. Jarrat, Some fourth order multipoint iterative methods for solving equations. Math. Comput. 20, 434-437 (1966).

[17] R.F. King, A family of fourth order methods for nonlinear equations. SIAM Journal of Numerical Analysis 10, 876-879 (1973).

[18] A. Cordero, J.L. Hueso, E. Martínez, J.R. Torregrosa, A modified Newton Jarratt's composition. Numerical Algorithms 55, 87-99 (2010).

[19] S. Amat, S. Busquier, Á. A. Magreñán, Reducing Chaos and Bifurcations in Newton-Type Methods. Abstract and Applied Analysis Volume 2013 (2013), Article ID 726701, 10 pages, http://dx.doi.org/10.1155/2013/726701.

[20] S. Amat, S. Busquier, S. Plaza, Review of some iterative root-finding methods from a dynamical point of view, Scientia Series A: Mathematical Sciences 10, 3-35 (2004).

[21] F. Chicharro, A. Cordero, J.M. Gutiérrez, J.R. Torregrosa, Complex dynamics of derivative-free methods for nonlinear equations, Applied Mathematics and Computation 219, 7023-7035 (2013).

[22] C. Chun, M.Y. Lee, B. Neta, J. Džunić, On optimal fourth-order iterative methods free from second derivative and their dynamics, Applied Mathematics and Computation 218, 6427-6438 (2012).

[23] Á. A. Magreñán, Different anomalies in a Jarratt family of iterative root-finding methods. Applied Mathematics and Computation 233, 29-38 (2014).

[24] A. Cordero, J.R. Torregrosa, P. Vindel, Dynamics of a family of Chebyshev-Halley type methods, Applied Mathematics and Computacion 219, 8568-8583 (2013).

[25] Á. A. Magreñán, Estudio de la dinámica del método de Newton amortiguado (PhD Thesis), Servicio de Publicaciones, Universidad de La Rioja, 2013. http://dialnet.unirioja.es/servlet/tesis?codigo=38821.

[26] P. Blanchard, The Dynamics of Newton's Method, Proc. of Symposia in Applied Math. 49, 139-154 (1994).

[27] F. Chicharro, A. Cordero, J.R. Torregrosa, Drawing dynamical and parameters planes of iterative families and methods, The Scientific World Journal Volume 2013 Article ID 780153.

[28] L.B. Rall, Computational Solution of Nonlinear Operator Equations, Robert E. Krieger Publishing Company, Inc., New York, 1969.

[29] J. R. Sharma, R. K. Guna and R. Sharma, An efficient fourth order weighted-Newton method for systems of nonlinear equations, Numerical Algorithms, 62 307-323 (2013). 